\title{
دراسة أحصائية حول تقدير المساحة المزروعة لمحصول الثلب في محافظة النجف المزرو
}

\author{
م. م. ماقبال محمود علوان الإدان \\ جامعة بغداد/ كلية الادارة والاقتصاد- \\ قسم الاحصاء
}

\begin{abstract}
The objective of the research is to find the best method to estimate rice crop through out evaluating the applied methods of stratified random sampling .By using different sorts of sampling estimators, a comparison was held among the variances of the mean for simple random sampling, stratified random sampling $\left(\operatorname{var}\left(\bar{y}_{s t}\right)\right)$ and separate regression estimator. The results indicate that the separate regression estimator give best estimations. The approximate cum. $f^{4 / 5}$ method was used to determine the optimum stratum boundaries, new strata was put and then var $\left(\bar{y}_{s t}\right)$ was calculated .In comparison with strata used nowadays in central statistical organization, the new strata led to obvious decrease in the variance. The stratified mean was estimated using the separate regression method for rice crop and comparing it with the population real estimate by testing $\chi^{2}$ between the real and estimate value.
\end{abstract}

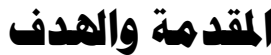

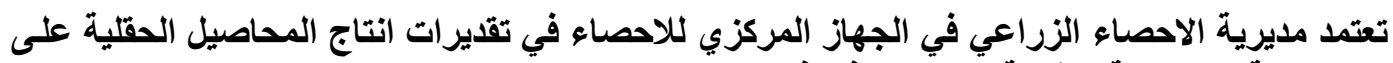

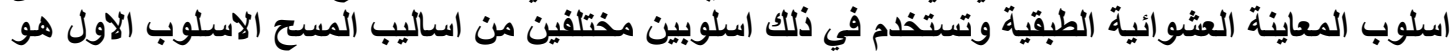

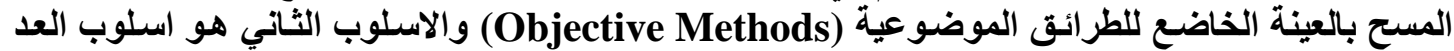

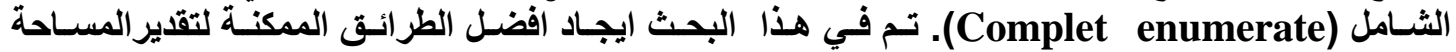

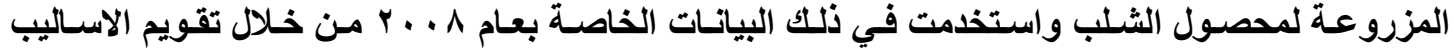

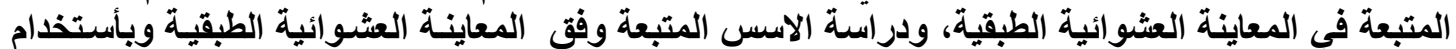

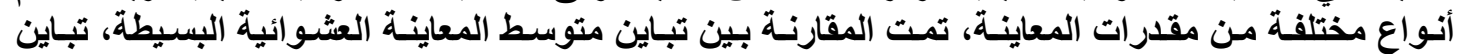

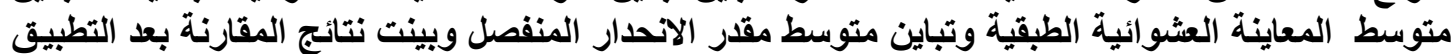

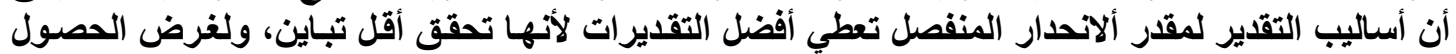

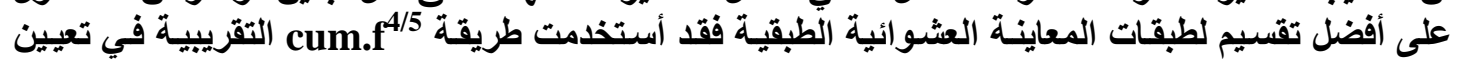

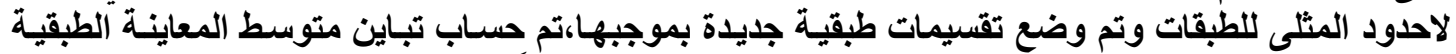

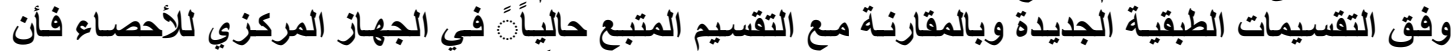

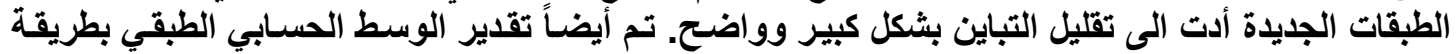

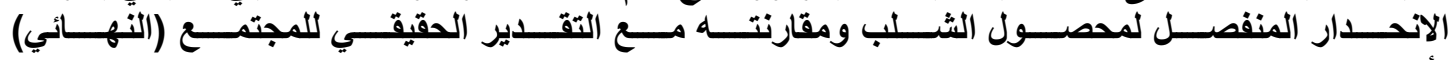
بأستخدام اختبار 20

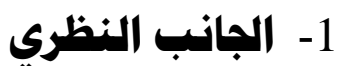

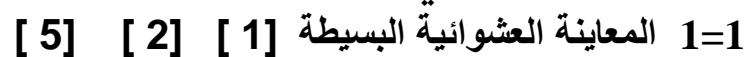
(Simple random Sampling :S.R.S)

$$
\bar{Y}=\frac{\sum_{i=1}^{N} Y_{i}}{N} \quad, \quad \bar{y}=\frac{\sum_{i=1}^{n} y_{i}}{n}
$$




$$
\begin{gathered}
\sigma^{2}=\frac{\sum_{i=1}^{N}\left(Y_{i}-\bar{Y}\right)^{2}}{N-1} \\
S^{2}=\frac{\sum_{i=1}^{n}\left(y_{i}-\bar{y}\right)^{2}}{n-1}
\end{gathered}
$$

وان تباين المجتمع يكتب بالصيغة:

وتباين العينة يكتب بالصيغة:

$$
\begin{aligned}
& \text { Y هو تقدير غير متحيز لمجموع المجتمع } \hat{Y}=N \bar{y}
\end{aligned}
$$

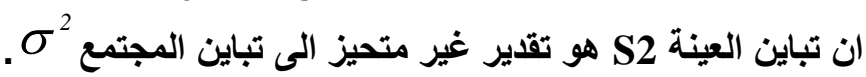

$$
\begin{aligned}
& \text { في العينة العشوائية البسيطة يكون تقدير التباين للمتوسط هو: } \\
& V(\bar{y})=\frac{S^{2}}{n} \cdot \frac{(N-n)}{N}=\frac{S^{2}}{n}(1-f)
\end{aligned}
$$

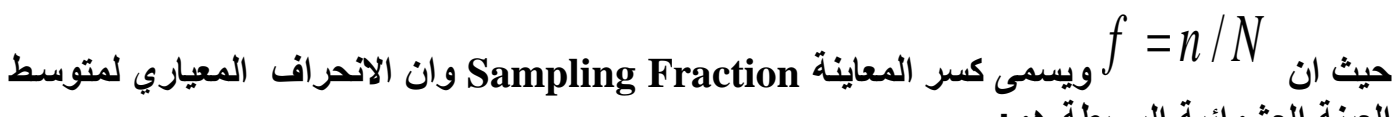
$\sigma_{y}=\frac{S}{\sqrt{n}} \sqrt{\frac{(N-n)}{N}}=\frac{S}{\sqrt{n}} \sqrt{1-f}$

$$
V(\hat{Y})=\frac{N^{2} S^{2}}{n} \cdot \frac{(N-n)}{N}=\frac{N^{2} S^{2}}{n}(1-f)
$$

ويكون تباين تقدير مجموع المجتمع:

\section{Stratified Random Sampling(ST.R.S.)}

\section{1-2 المعاينة العشوائية الطبقبة [1 ] [6 ] [7 ].}

ان دقة التقدير او تباين المتوسط مجتمع تتوقف على حجم العينة. وفي المعاينـة الطبقيـة يتم تقسيم

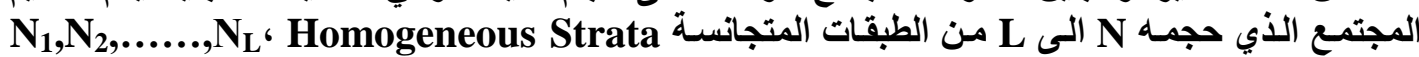

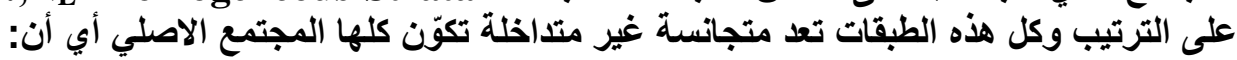
$N=\sum_{h=1}^{L} N_{h}=N_{1}+N_{2}+\ldots . .+N_{L}$

ثم نقوم بسحب عينات عشوائية بسيطة داخل الطبقات أحجامها n1,n2,.....,n على التوالي بحيث $n=\sum_{h=1}^{L} n_{h}=n_{1}+n_{2}+\ldots \ldots+n_{L}$

سنفرض أن عدد وحدات المجتمع N وأن عدد طبقات المجتمع هو L ولنأخذ الطبقة h ونـرمز للوحدة

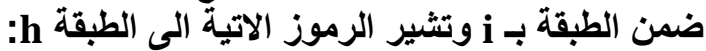

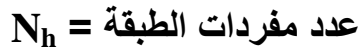
$h=1,2, \ldots . ., L$ 


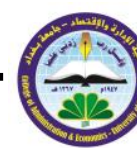

عل مدرد مفردات العينة المسحوبة من الطبقة =

$$
\begin{gathered}
W_{h}=N_{h} / N \\
f_{h}=n_{h} / N_{h}
\end{gathered}
$$

$Y_{h 1}, Y_{h 2}, \ldots \ldots, Y_{h N_{h}}$

$y_{h 1}, y_{h 2}, \ldots \ldots, y_{h n_{h}}$
وزن (ترجيحة) الطبقة

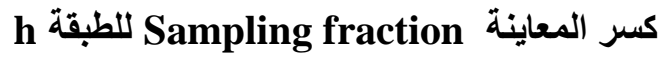

مفردات المجتمع للطبقة h هي:

مفردات العينة للطبقة $\mathrm{h}$ هي :

القيمة التي نحصل عليها في الوحدة (i) من الطبقة

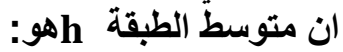

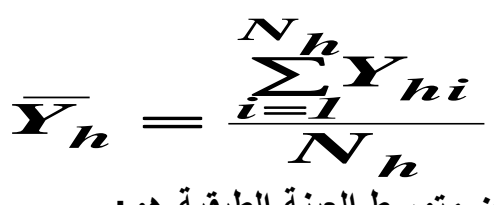

أن متوسط العينة الطبقية هو:

$\bar{y}_{h}=\frac{\sum_{i=1}^{n_{h}} y_{h i}}{n_{h}}$

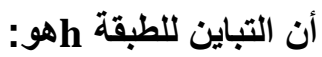

$\sigma_{h}^{2}=\frac{\sum\left(y_{h i}-\overline{Y_{h}}\right)^{2}}{N_{h}-1}$

وان تباين العينة للطبقة هو h

$S_{h}^{2}=\frac{\sum_{i=1}^{n h}\left(y_{h i}-\bar{y}_{h}\right)^{2}}{n_{h}-1}$

$V\left(\bar{y}_{h}\right)=S_{h}^{2}\left(1-f_{h}\right) / n_{h}$

أن تباين متوسط العينة الطبقية هو:

متوسط المعاينة الطبقية

$$
\bar{y}_{s t}=\sum_{h=1}^{L} W_{h} \bar{y}_{h}
$$

$\bar{y}_{s t}=\frac{\sum_{h=1}^{L} N_{h} \bar{y}_{h}}{N}$ 


$$
N=N_{1}+N_{2}+\ldots \ldots+N_{L}
$$

اذا كان الوسط الحسابي لعينات الطبقات

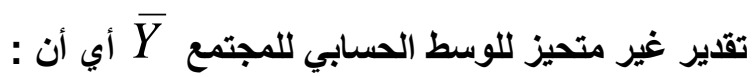
$E \bar{y}_{s t}=\bar{Y}=\sum_{h=1}^{L} W_{h} \bar{Y}_{h}$

إن تباين متوسط المعاينة الطبقية يكون حسب الصيغةالآتية:

$$
\begin{aligned}
& V\left(\bar{y}_{s t}\right)=\sum_{h=1}^{L} W_{h}^{2} V\left(\bar{y}_{h}\right) \\
& V\left(\bar{y}_{s t}\right)=\frac{1}{N^{2}} \sum_{h=1}^{L} N_{h}\left(N_{h}-n_{h}\right) \frac{S_{h}^{2}}{n_{h}} \\
& =\sum_{h=1}^{L} W_{h}^{2} \frac{S_{h}^{2}}{n_{h}}\left(1-f_{h}\right) .
\end{aligned}
$$

واذا كان

$$
V\left(\hat{Y}_{s t}\right)=\sum_{h=1}^{L} N_{h}\left(N_{h}-n_{h}\right) \frac{S_{h}^{2}}{n_{h}}
$$

حيث ان تباين

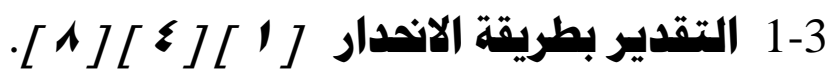

\section{Regression Estimate}

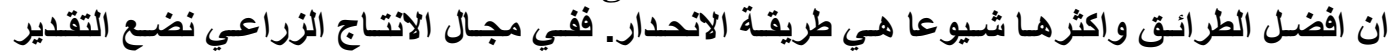

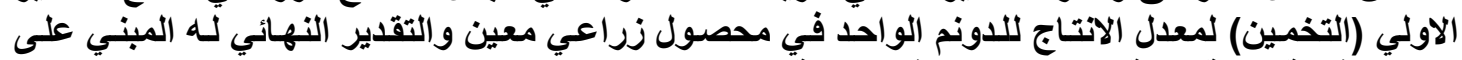

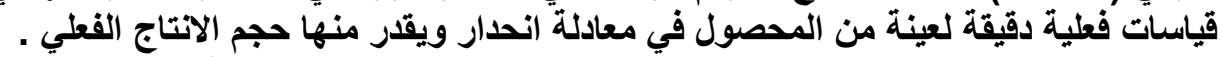

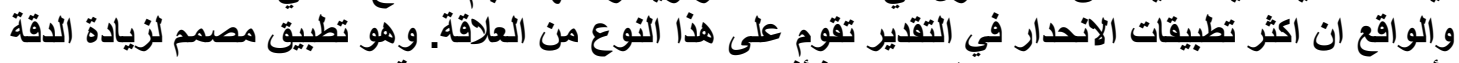

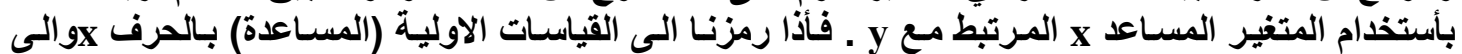

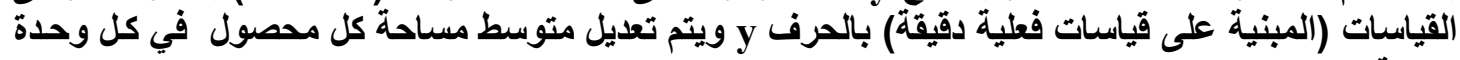

$$
\bar{y}_{l r}=\bar{y}-b(\bar{X}-\bar{x})
$$

معاينة بمقدر الاتحدارالاتي : عل :

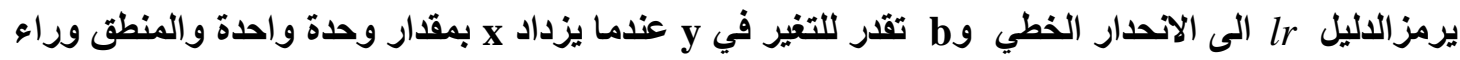
هذا التقدير هو انه اذال كان

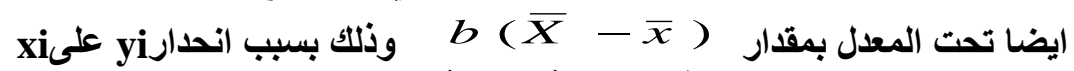
من انواع مقدرات الاتحدار في المعاينة الطبقية هو: 
Separate Regression estimator 1-3-1 هقدر الانشدار المنفصل يرمز لتقير الوسط الحسابي الطبقي المنفصل بطريقة الانحار بالرمز

$$
\bar{y}_{l l h}=\bar{y}_{h}+b_{h}\left(\bar{X}_{h}-\bar{x}_{h}\right)
$$

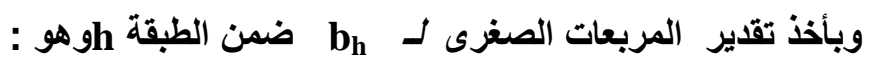

$$
\begin{aligned}
b_{h} & =\frac{\sum_{i}\left(y_{h i}-\bar{y}_{h}\right)\left(x_{h i}-\bar{x}_{h}\right)}{\sum_{i}\left(x_{h i}-\bar{x}_{h}\right)^{2}} . \\
\bar{y}_{l r s} & =\sum_{h=1}^{l} W_{h} \bar{y}_{l r h} \ldots \ldots \ldots \ldots \ldots \ldots
\end{aligned}
$$

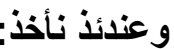

ويكون هذا التقدير مناسب عندما يختلف معامل الاتحار الحقيقي bن من طبقة الى طبقة . وان تباين المتوسط لهذا المقدر هو :

$$
\operatorname{var}\left(\bar{y}_{l s}\right)=\sum_{h=1}^{l} \frac{W_{h}^{2}}{n_{h}} \frac{\left(1-f_{h}\right)}{n_{h}}\left[\frac{1}{n_{h}-2}\right]\left[\sum_{i}^{n h}\left(y_{h i}-\bar{y}_{h}\right)^{2}-b_{h}^{2} \sum_{i}^{h}\left(x_{h i}-\bar{x}_{h}\right)^{2}\right] \ldots
$$

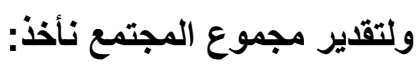

$$
\begin{aligned}
& {\widetilde{Y_{l r s}}}=N \bar{y}_{l r s} \cdots \\
& {\widetilde{Y_{l r s}}}=N \sum_{h=1}^{l} W_{h} \bar{y}_{l r h}
\end{aligned}
$$

$$
V\left(\widetilde{Y_{l r s}}\right)=N^{2} V\left(\bar{y}_{l r h}\right)
$$

$$
V\left(\hat{Y_{l r s}}\right)=\sum_{h=1}^{L} N_{h}\left(N_{h}-n_{h}\right) \frac{S_{h}^{2}}{n_{h}}
$$

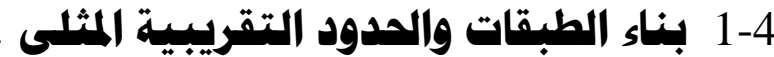

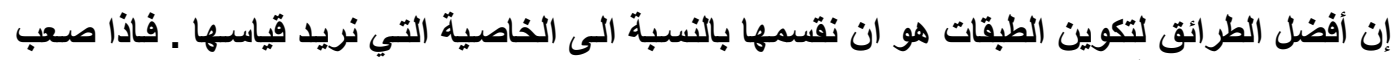

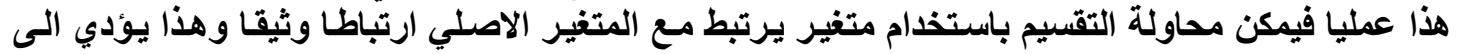
تصغير التباين . ت فيكن

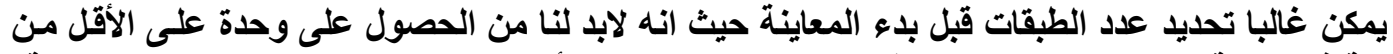

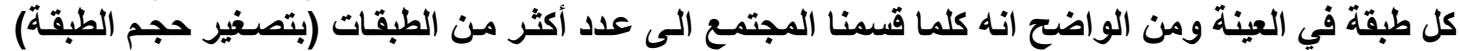

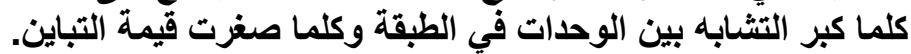

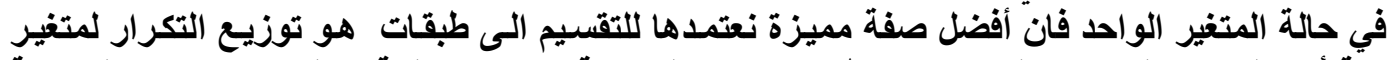

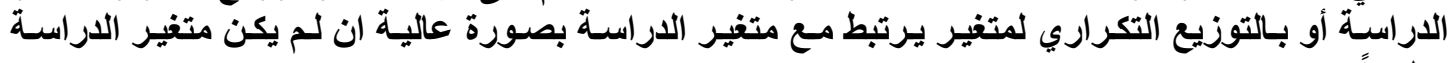




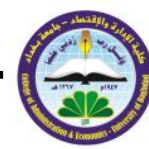

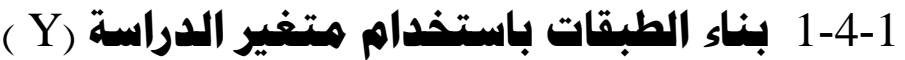

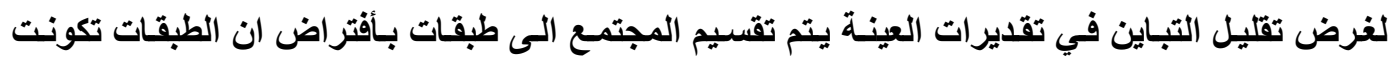

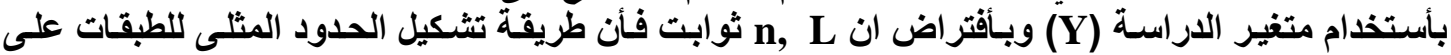

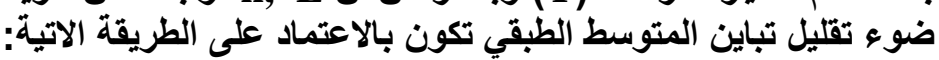

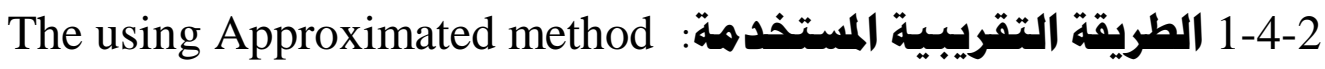
طum.f لنفرض أنه تم تقسيم المجتمع قيد الاراسة الى L من الطبقات وتم سحب عينة عشوائية بسيطة من كل

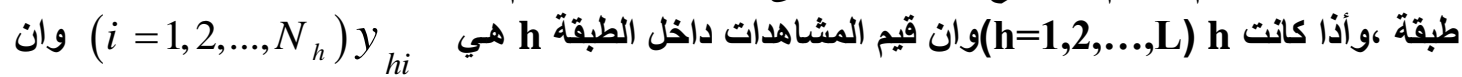

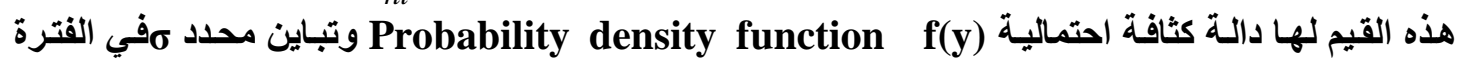
: $[\mathbf{a}, \mathbf{b}]$

$$
\begin{aligned}
& z(y)=\int^{b} f^{4 / 5}(y) d y \\
& \text { وبتجزئة } \\
& Z_{h}(y)=\int_{y_{h-1}}^{y_{h}} f^{4 / 5}(y) d y \\
& h=1,2, \ldots, L-1
\end{aligned}
$$

وبما ان بو هو المتوسط التقريبي لـf(y) داخل الطبقة h فان :

$Z_{h}(y)=\int_{y_{h-1}}^{y_{h}} \mu_{h}^{4 / 5} d y=\mu_{h}^{4 / 5}\left(y_{h}-y_{h-1}\right)$

$$
\mu_{h}^{4 / 5}=\frac{Z_{h}(y)}{\left(y_{h}-y_{h-1}\right)}
$$$$
\mu_{h}=\left[\frac{Z_{h}(y)}{\left(y_{h}-y_{h-1}\right)}\right]^{5 / 4} \ldots
$$

أن تباين المتوسط الطبقي باستذام التوزيع الأمثل عند إهمال كسر المعاينة هو:

$$
\mathrm{V}_{\mathrm{opt}}\left(\overline{\mathrm{y}}_{\mathrm{st}}\right)=\frac{1}{\mathrm{n}}\left(\sum_{\mathrm{h}=1}^{\mathrm{L}} \mathrm{W}_{\mathrm{h}} \sigma_{\mathrm{h}}\right)^{2}
$$$$
V_{o p t}\left(\bar{y}_{s t}\right)=\frac{1}{n}\left[\sum_{h=1}^{L} M_{h}\left(y_{h}-y_{h-1}\right) \frac{\left(y_{h}-y_{h-1}\right)}{(12)^{1 / 2}}\right]^{2}
$$

وبالتعويض عن قيمة $\sigma_{\mathrm{h}}, \mu_{\mathrm{h}} \textrm{ }$ 


$$
\begin{aligned}
& V_{\text {opt }}\left(\bar{y}_{s t}\right)=\frac{1}{12 n}\left[\sum_{h=1}^{L} \frac{\mathrm{Z}_{h}{ }^{2}(y)}{\left(y_{h}-y_{h-1}\right)^{2}}\left(y_{h}-y_{h-1}\right)^{2}\right]^{2} \\
& =\frac{1}{12 n}\left[\sum_{h=1}^{L} Z_{h}{ }^{5 / 2}(y)\right] \ldots \ldots \ldots \ldots \ldots \ldots \ldots \ldots \ldots
\end{aligned}
$$

ان المعادلة السـابقة تصبح أقل مـا يمكن عندما تكون Zh (y) ثابتا لجميع قيم h وذلكك باستخدام مضـاعف لاكرانج تحت القيد (y)

$Z_{h}(y)=\frac{Z(y)}{L}$

$$
V_{o p t}\left(\bar{y}_{s t}\right)=\frac{Z^{5 / 2}(y)}{12 n L^{2}}
$$

وان تباين المتوسط الطبقي في هذه الحالة يصبح :

اذ ان تباين متوسط المعاينة الطبقية للطريقة التقريبية Cum.f يكون باستخدام القاعدة العامة التالية :

$$
V_{o p t}\left(\bar{y}_{s t}\right)=\frac{Z^{2 n / m}(y)}{12 n L^{2}}
$$

$Z(y)=\int_{y_{h-1}}^{y_{h}} f^{m / n} d y$

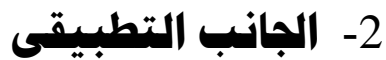

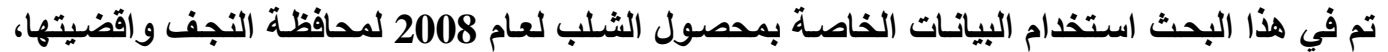

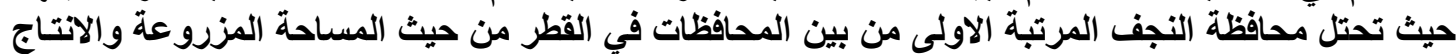

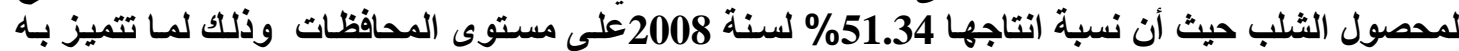
هذه المحافظة من تربة مناسبة لزراعته أنتاجية

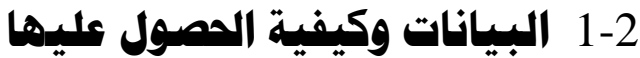

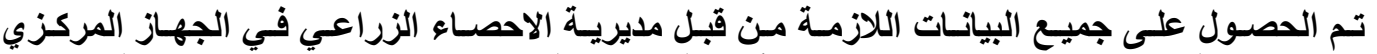

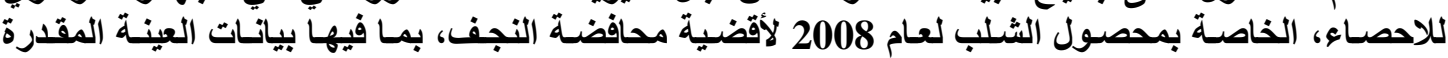
والفطية* للمساحة المزروعة لكل حائز بالدونم على مستوى الاقضية حيث ان محافظة النجف تثألف من ثلاثل

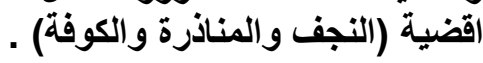




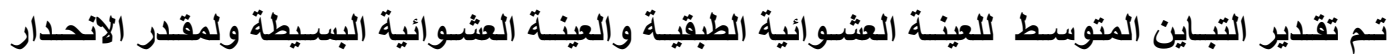

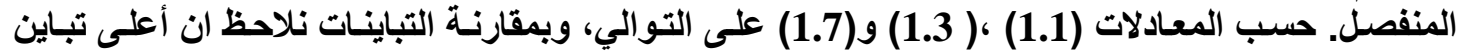

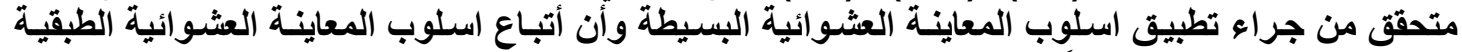

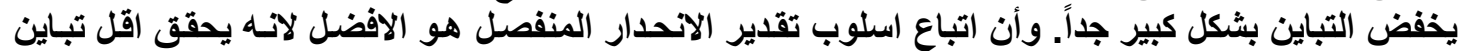

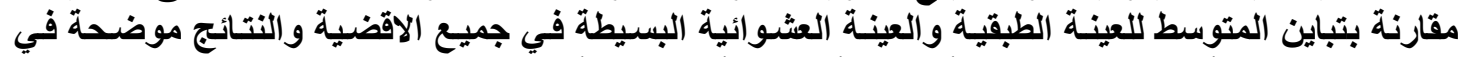
الجدول رقم (1) وأن جميع التطبيقات أجريت بأستخدام ألبرنامج الأحصائي Minitab 11 for Windows.

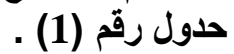

تباين المتوسط للعينة العشوائية الطبقية، البسيطة ولمقدرالانحدار المنفصل.

\begin{tabular}{|c|c|c|c|}
\hline المناذرة & الكوفة & النجف & القضاء \\
\hline 1717.7833 & 367.2138 & 10.4192 & $\operatorname{Var}(\bar{y})$ \\
\hline 0.6279 & 0.2056 & 0.0073 & $\operatorname{Var}\left(\bar{y}_{s t}\right)$ \\
\hline 0.0815 & 0.0861 & 0.0072 & $\operatorname{Var}\left(\bar{y}_{l r s}\right)$ \\
\hline
\end{tabular}

2-3 بناء الطبقات والهدود المثلى للطبقات

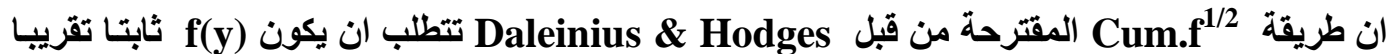

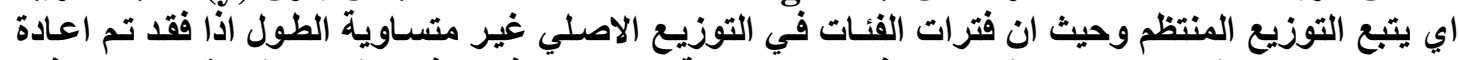

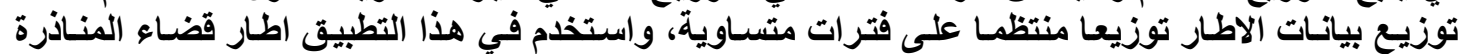

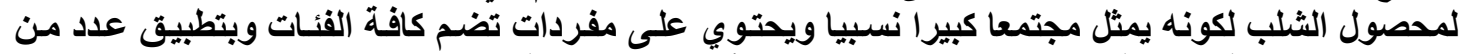

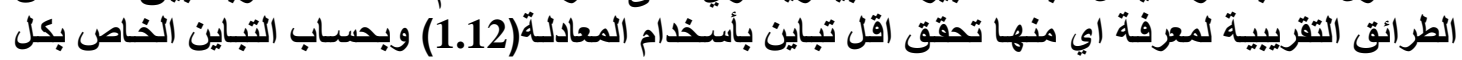

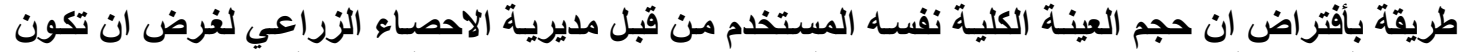

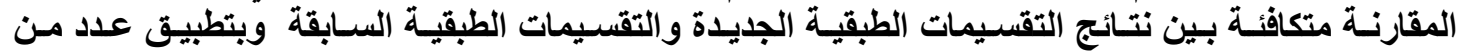

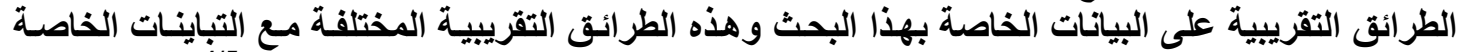

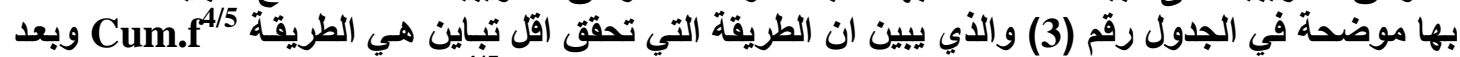

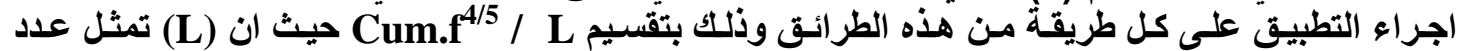

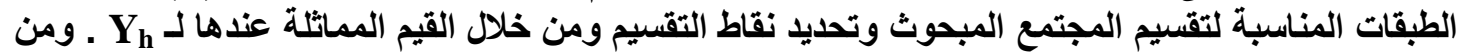

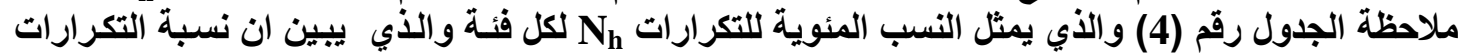

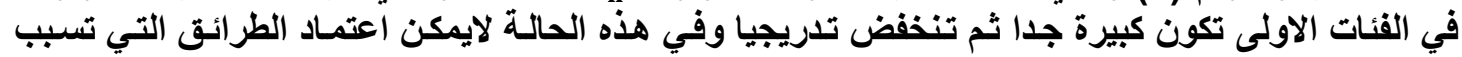

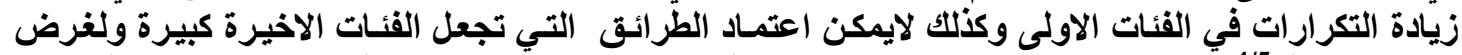

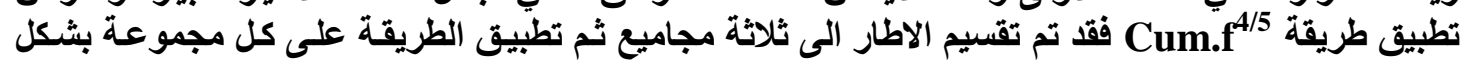

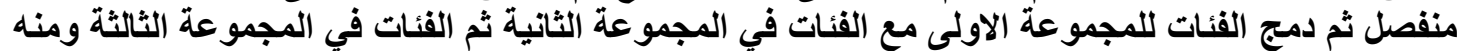

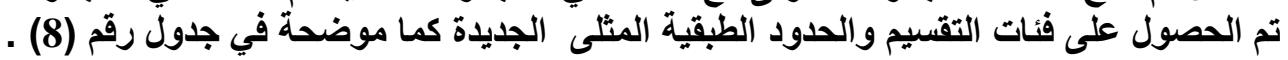


جدول رقم (2) الطر ائق التقريبية المختلفة

\begin{tabular}{|c|c|c|c|}
\hline $\mathbf{F}(\mathbf{y})^{2 \mathrm{n} / \mathrm{m}}$ & $F(y)$ & الطريقة Cum.f & التسلسل \\
\hline$F(y)^{6}$ & 103.4360 & Cum.f $^{2 / 6}$ & 1 \\
\hline$F(y)^{4}$ & 272.8155 & Cum.f $^{1 / 2}$ & 2 \\
\hline$F(y)^{7 / 2}$ & 416.4380 & Cum.f $^{4 / 7}$ & 3 \\
\hline$F(y)^{3}$ & 757.3520 & Cum.f.$^{2 / 3}$ & 4 \\
\hline$F(y)^{8 / 3}$ & 8940.1220 & Cum.f $^{3 / 4}$ & 5 \\
\hline$F(y)^{5 / 2}$ & 1651.5212 & Cum.f $^{4 / 5}$ & 6 \\
\hline
\end{tabular}

$V_{o p t}\left(\bar{y}_{s t}\right)$ جدول رقم (3) الطرائق التقريبية Cum.f مع تبايناتها

\begin{tabular}{|c|c|}
\hline$V_{\text {opt }}\left(\bar{y}_{s t}\right)$ & Cum.f $^{\text {m/n }}{ }^{\text {12599811.2700 }}$ \\
\hline $\mathbf{5 6 9 9 1 . 4 7 5 3}$ & Cum.f $^{2 / 6}$ \\
\hline 15162.1213 & Cum.f $^{1 / 2}$ \\
\hline 4469.1719 & Cum.f $^{4 / 7}$ \\
\hline 1751.7610 & Cum.f $^{2 / 3}$ \\
\hline 1140.4153 & Cum.f $^{3 / 4}$ \\
\hline
\end{tabular}

جدول رقم (4) النسب المئوية لحجم كل فئة لمحصول الثلب 2008

\begin{tabular}{|c|c|c|}
\hline النسبة المئوية & حدود الفئة (الطبقة) & الفئة (الطبقة) \\
\hline$\% 22.8000$ & اقل من 6 & الفئة الاولى \\
\hline$\% 42.8700$ & 6 واقل من 12 & الفئة الثانية \\
\hline$\% 23.6500$ & 12 واقل من26 & الفئة الثالثة \\
\hline$\% 6.2400$ & 26 واقل من51 & الفئة الرابعة \\
\hline$\% 2.4200$ & 51 واقل من 101 & الفئة الخامسة \\
\hline$\% 1.3600$ & 101 واقل من 201 & الفئة السادسة \\
\hline$\% 0.5100$ & 201 واقل من 401 & الفئة السابعة \\
\hline$\% 0.1000$ & 401 واقل من 1001 & الفئة الثامنة \\
\hline$\% 0.0300$ & 1001 واقل من 2000 & الفئة التاسعة \\
\hline
\end{tabular}

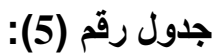

نتائج تقسيم Cum.f على قيم مختلفة لـ (L) لاطار قضاء المناذرة لمحصول الثلب

\begin{tabular}{|r|c|}
\hline L & Cum.f $^{4 / 5} / \mathrm{L}$ \\
\hline 3 & 0.5071 \\
\hline 4 & $\mathbf{0 . 8 8 0 3}$ \\
\hline 5 & $\mathbf{0 . 3 0 4 2}$ \\
\hline 6 & 0.2535 \\
\hline
\end{tabular}

2-4 التقسيسمات وهدود الطبقات الاقتزمة

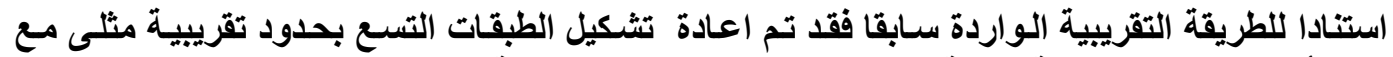

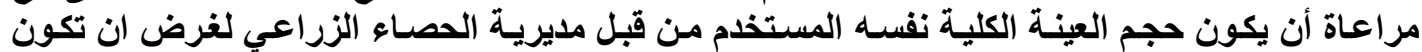

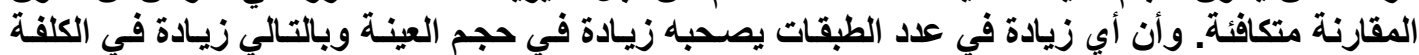

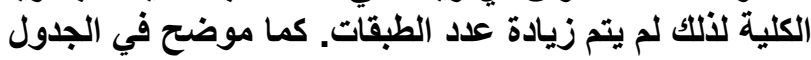


جدول رقم (6) التقسيم الجديد لحدود الفئات

\begin{tabular}{|c|c|}
\hline حدود الفئة (الطبقة) & الفئة (الطبقة) \\
\hline اقل من 8 & الفئة الاولى \\
\hline 8 واقل من 14 & الفئة الثانية \\
\hline 14 واقل من 32 & الفئة الثالثة \\
\hline 32 واقل من 52 & الفئة الرابعة \\
\hline 52 واقل من 92 & الفئة الخامسة \\
\hline 92 واقل من 202 & الفئة السادسة \\
\hline 202 واقل من 302 & الفئة السابعة \\
\hline 302 واقل من 402 & الفئة الثامنة \\
\hline 402 واقل من 5000 & الفئة التاسعة \\
\hline
\end{tabular}

واستنادا الى هذه الطبقات والحدود الطبقيـة المثلى الجديدة للفئـات فقد تم اعـادة تقسيم بيانـات الاطر

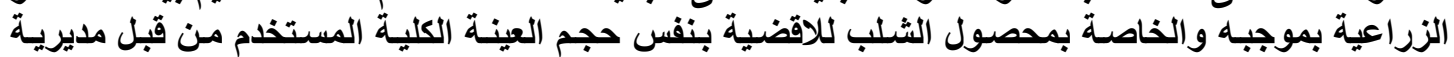

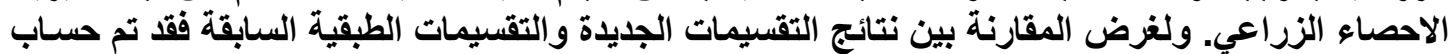

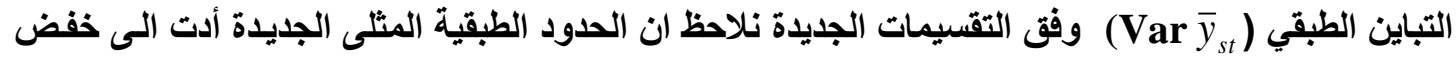
التباين للاقضية كافة بثكل واضح وكبير،كما موضح في الجدول التالي :

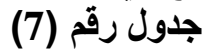

التباين الطبقي (Var $\left(\bar{y}_{s t}\right.$ وفق الحدود الطبقية المثلى الجديدة لفئات اقضية محافظة النجف .

\begin{tabular}{|c|c|}
\hline $\operatorname{Var}\left(\bar{y}_{s t}\right)$ & القضاء \\
\hline $\mathbf{0 . 1 1 5 0}$ & المناذرة \\
\hline 0.1205 & الكوفة \\
\hline 0.0072 & النجف \\
\hline
\end{tabular}

2-5 تقدير الانصدار المنفصل

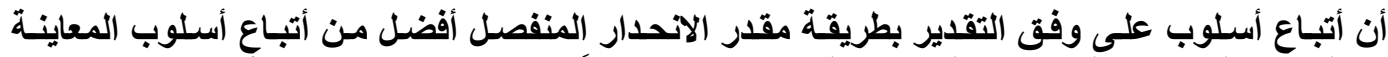

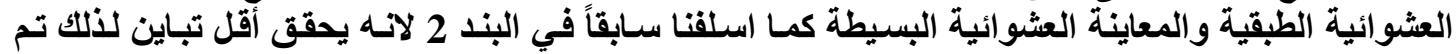

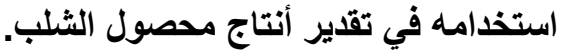

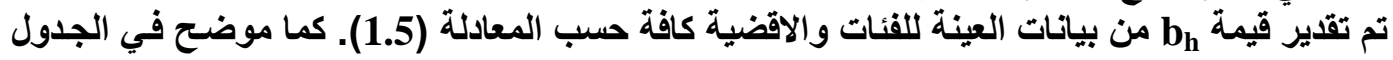
جدول رقم (8) قيم bh للفئات (الطبقات) والاقضية لمحافظة النجف كافة.

\begin{tabular}{|c|c|c|c|c|}
\hline \multicolumn{3}{|c|}{$\mathbf{b}_{\mathrm{h}}$} & \multirow{2}{*}{ حدود الفئة (الطبقة) } & \multirow{2}{*}{ الفئة (الطبقة) } \\
\hline قضاء الكوفة & قضاء المناذرة & قضاء النجف & & \\
\hline $\mathbf{0 . 7 6 3 0}$ & 0.9220 & 0.2300 & اقل من 6 & الفئة الاولى \\
\hline 1.0880 & 0.8450 & 0.1300 & 6 و واقل من 12 & الفئة الثانية \\
\hline 1.0070 & 1.2220 & 0.0280 & 12 واقلّ من26 & الفئة الثالثة \\
\hline 0.6070 & 1.0250 & 0.2280 & 26 واقلّ من51 & الفئة الرابعة \\
\hline 0.0070 & 1.3370 & & 51 واقل من 101 & الفئة الخامسة \\
\hline 0.9890 & 1.0170 & & 101 واقل من 201 & الفئة السادسة \\
\hline 1.0220 & 0.8290 & & 201 واقل من 401 & الفئة السابعة \\
\hline 0.9140 & $\mathbf{0 . 7 0 3 0}$ & & 401 واقلّ من 1001 & الفئة الثامنة \\
\hline 0.000 & 0.7960 & & 1001 و اقل من 2000 & الفئة التاسعة \\
\hline
\end{tabular}

كما اظهرت نتائج التطبيق ان قيم bخت bخة من طبقة الى اخرى لذا تم استخدام مقدار الاتحدار المنفصل. 
تم حساب الانحدار المتوسط Irh لكل فئة (طبقة) حسب المعادلة (1.4) كما في الجدول التالي:

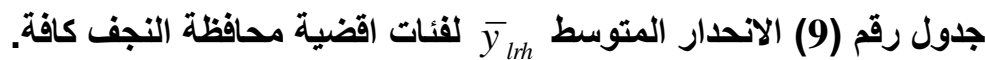

\begin{tabular}{|c|c|c|c|}
\hline \multicolumn{3}{|c|}{$\bar{y}_{\text {Irh }}$} & \multirow{2}{*}{ الفئة (الطبقة) } \\
\hline الكوفة & المناذرة & النجف & \\
\hline 4.5803 & 4.2601 & 2.9800 & الفئة الاولى \\
\hline 9.0711 & 7.6052 & 6.3300 & الفئة الثانية \\
\hline 18.2790 & 15.5700 & 19.0400 & القئة الثالثة \\
\hline 30.0590 & $\mathbf{3 7 . 2 9 3 3}$ & 27.7600 & الفئة الرابعة \\
\hline 73.6900 & 93.2900 & & الفئة الخامسة \\
\hline 143.3830 & 163.8030 & & الفئة السادسة \\
\hline 306.4930 & 279.9145 & & الفئة السابعة \\
\hline 450.9600 & $52^{r} .1800$ & & الفئة الثامنة \\
\hline 1354.2200 & 2911.0000 & & الفئة التاسعة \\
\hline
\end{tabular}

تم حساب الوسط الحسابي الطبقي المنفصل Irs حسب المعادلة (1.6) كما في الجدول التالي: جدول رقم (10) الوسط الحسابي الطبقي الأقضية محافظة النجف كافة .

\begin{tabular}{|c|c|}
\hline $\bar{y}_{\mathbf{I r s}}$ & القضاء \\
\hline 9.4780 & النجف \\
\hline 13.6134 & الكوفة \\
\hline 17.2100 & المناذرة \\
\hline
\end{tabular}

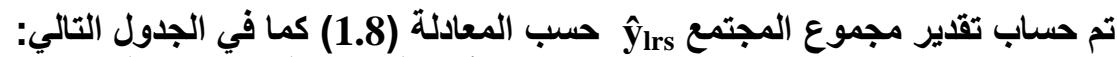

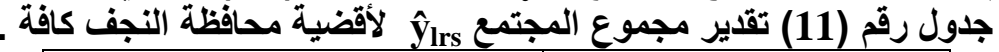

\begin{tabular}{|c|c|}
\hline$\hat{\mathbf{y}}_{\text {Irs }}$ & القضاء \\
\hline 1279.5300 & النجف \\
\hline 64690.8760 & الكوفة \\
\hline 108612.3100 & المناذرة \\
\hline
\end{tabular}

جدول رقم (12) القيم الفعلية والتقديرية والفروق المطلقة بينهما والنسب المئوية

للفروق المطلقة لمحصول الثلب لعام 2008

\begin{tabular}{|c|c|c|c|c|}
\hline اللفبة المئوية & الفروق المطلقة & المساحة التقديرية & المساحة الحقيقية & القضاء \\
\hline $0.40 \%$ & 5.1350 & 1279.5300 & 1284.6650 & النجف \\
\hline $0.11 \%$ & 70.6368 & 64690.8760 & 64620.2400 & الكوفة \\
\hline $0.82 \%$ & 878.0500 & 108612.3100 & 107734.2600 & المناذرة \\
\hline
\end{tabular}




\section{r-1 اختبار معنوية الفرق بين القيم الهقيقية والقيم التقديرية .}

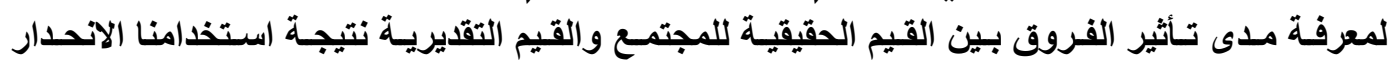

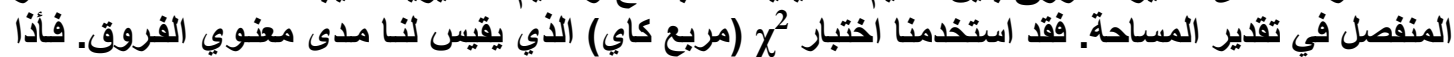

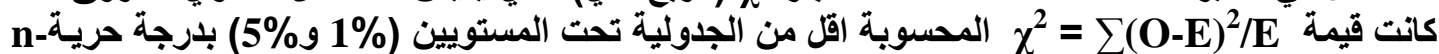

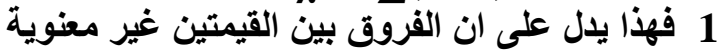

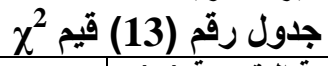

\begin{tabular}{|c|c|c|c|c|c|}
\hline$(\mathbf{O}-\mathbf{E})^{2} / \mathbf{E}$ & $(\mathrm{O}-\mathrm{E})^{2}$ & O-E & المساحة التقديرية (E) & المساحة الحقيقية (O) & القضاء \\
\hline 0.0210 & 26.3680 & 5.1350 & 1279.5300 & 1284.6650 & النجف \\
\hline 0.0774 & 5004.1334 & 70.6368 & 64690.8760 & 64620.2400 & الكوفة \\
\hline 7.1518 & 770971.8025 & 878.0500 & 107800.2500 & 107734.2600 & المناذرة \\
\hline
\end{tabular}

$$
\begin{gathered}
\chi^{2}=\sum(\mathrm{O}-\mathrm{E})^{2} / \mathrm{E}=7.25 \\
\chi_{(0.01 \times 2)}^{2}=5.991 \\
\chi_{(0.05 ، 2)}^{2}=9.210
\end{gathered}
$$

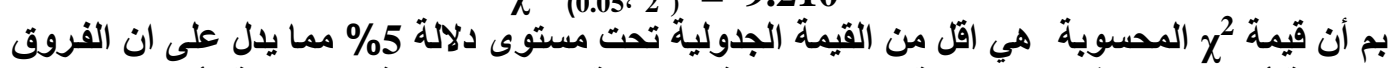

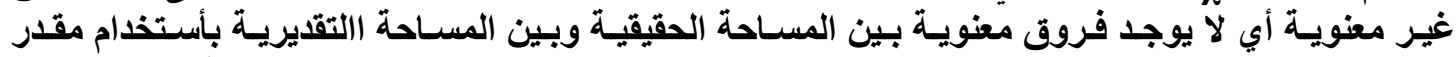
الانحدار المنفصل، وهذا يؤدي اللى دعم اسخدام الانحدار المنفصل في تقدير المتيل المحاصيل الزراعية. 
2-7 الإستنتاجات

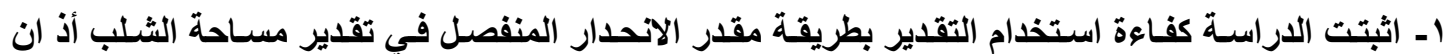

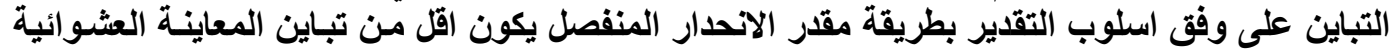

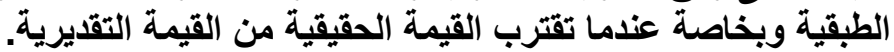

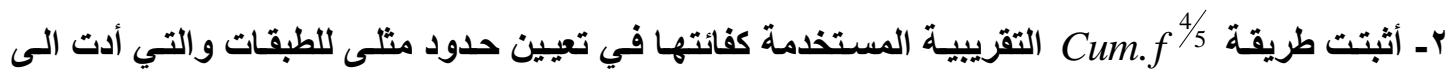
تقليل التباين بشكل كبيز وواضح .

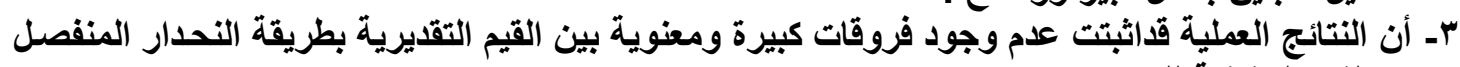
والقيم الحقيقية للمجتمع. تصأبتع

2-8 بناءا على الإستنتاجات الواردة سابقاً فإن البحث أعطى التوصيات الآتية:

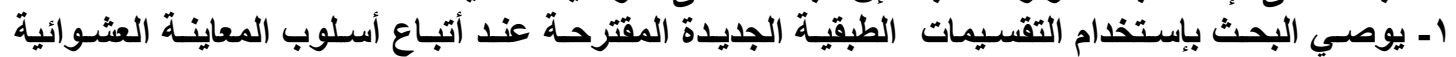

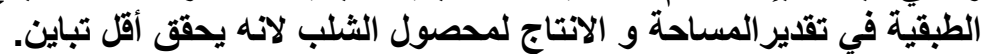

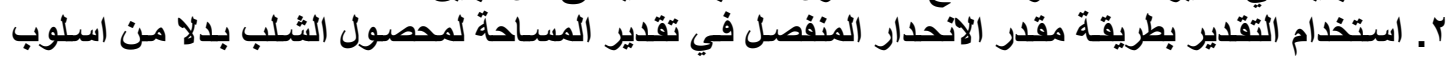

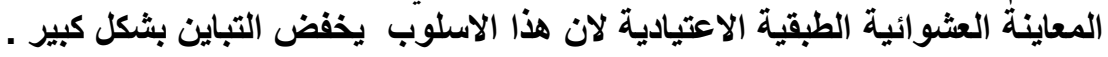
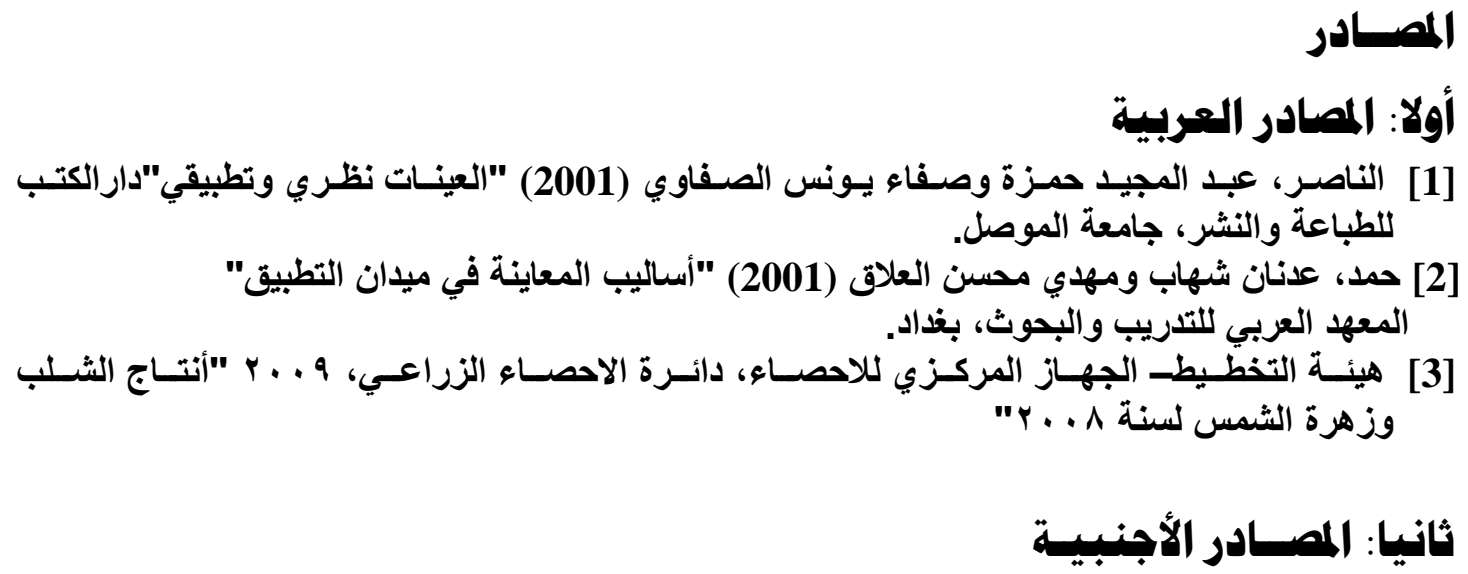

[4] Cochran W. G. (1977) "Sampling Techniques", $3^{\text {rd }}$ Edition,New York:John Wiley and Sons.

[5] Jones, H. L.,(1968) "The analysis of Variance of Data from Stratiefied Subsample " JASA ,63,pp.64-86.

[6] Konijn ,H. S.(1973) "Statistical Theory of Sample Survey Design and analysis" North -Holland publishing company-Amsterdam.

[7] Shapiro ,G.m.\& Olsen, C. L.(1979) "Should one or two Psu's ser stratum be selected?" Amer .Statistical Assn .1979 proceeding of the section on Survery Reserch Methods,pp314-318.

[8] Yates, F.|(1960) "Sampling methods for censues and surveys" Charles Grffin Co.,Ltd.,London . 